\title{
Inter-rater reliability of a clinical staging of HIV-associated cognitive impairment
}

\author{
K. Marder, MD, MPH; S.M. Albert, PhD; M.P. McDermott, PhD; J.C. McArthur, MBBS, MPH; \\ G. Schifitto, MD; O.A. Selnes, PhD; N. Sacktor, MD; Y. Stern, PhD; D. Palumbo, PhD; \\ K. Kieburtz, MD, MPH; B. Cohen, MD; C. Orme BA; and L.G. Epstein, MD
}

\begin{abstract}
Objective: To determine the inter-rater reliability of a modification of the Memorial Sloan-Kettering (MSK) Staging for HIV-associated cognitive impairment. Methods: Data were abstracted on neurologic, neuropsychological, and functional status on 100 individuals participating at four sites in the Northeast AIDS Dementia (NEAD) Consortium cohort study, a longitudinal study of predictors of cognitive impairment in HIV-infected individuals. Neuropsychological performance was defined 1) based on the neuropsychologist's global impression and 2) solely based on neuropsychological test scores. Raters at each site used the abstracted data to assign an MSK stage to each subject blind to any identifying information. Inter-rater reliability was assessed using kappa statistics. Agreement between computer-generated ratings and site-generated ratings was also assessed. Results: Kappa statistics for pair-wise agreement among the sites regarding MSK stage ranged from 0.70-0.91, representing good to excellent agreement between sites. Agreement between computer-generated ratings and site-generated ratings was in the good to excellent range (0.62-0.79). Conclusions: The authors have modified the MSK rating scale and developed a reliable instrument that can be used in multicenter studies. This instrument will be useful in staging HIV-dementia in future longitudinal studies and will be valuable in increasing accuracy of clinicopathologic studies.
\end{abstract}

NEUROLOGY 2003;60:1467-1473

In February 1998, the Northeast AIDS Dementia (NEAD) Consortium was created to recruit a cohort of individuals at high risk for the development of HIV-dementia (HIV-D). This cohort was initially established at three sites: Columbia University, Johns Hopkins University, and the University of Rochester. In 1999, a site at Northwestern University was added. Inclusion and exclusion criteria for this new cohort were identical to those used in a previous collaboration, the Dana Consortium on Therapy for HIV Dementia and Related Cognitive Disorders, established in February 1994. ${ }^{1}$

One of the primary aims of the Dana Consortium was to determine predictors of the development of HIV-D, including HIV-Associated Minor Cognitive Motor Disorder (MCMD). To uniformly diagnose both HIV-D and MCMD across sites, we developed a computerized algorithm to operationalize the criteria proposed by the American Academy of Neurology in 1991. ${ }^{2}$ These operationalized criteria were then used to define HIV-D and MCMD in 272 individuals in the Dana cohort. ${ }^{1}$ In longitudinal analyses, over a follow-up period of up to 30 months, we examined factors associated with time to develop HIV-D. ${ }^{3}$

The Memorial Sloan Kettering (MSK) Staging of the AIDS dementia complex ${ }^{8}$ was devised in 1988 in order to "establish a common vocabulary for epidemiologic, natural history, and therapy studies with criteria that are reasonably easy to establish based on the clinical examination and that do not require special expertise in neuropsychological testing." It has been used in clinical trials ${ }^{4-7}$ and in a cohort study. ${ }^{1}$ The stages from 0 (normal) to 4 (end stage) are differentiated primarily by degree of functional impairment in activities including work and self-care and also by mobility status. Details on specific neurologic and neuropsychological abnormalities are not specifically delineated. In an attempt to standardize the interpretation of these ratings, we operationalized the definitions of each MSK stage using information from neurologic and neuropsychological examinations and functional assessments and then assessed the inter-rater reliability of this algorithm across four sites participating in the NEAD cohort.

Subjects. To be included in the NEAD cohort, subjects had to be HIV-positive, and have either a $\mathrm{CD}^{+}$cell count below $200 / \mu \mathrm{L}$ or a $\mathrm{CD} 4^{+}$count below $300 / \mu \mathrm{L}$ with cognitive impairment on a neuropsychological test battery. Exclusion criteria were 1) current or past opportunistic CNS infection at study entry with the exception of treated neurosyphilis prior to diagnosis of HIV, 2) history or current clini-

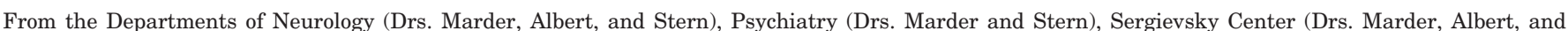

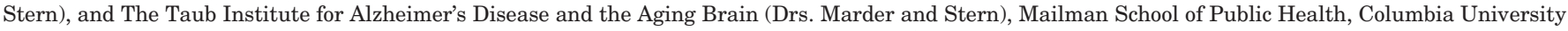

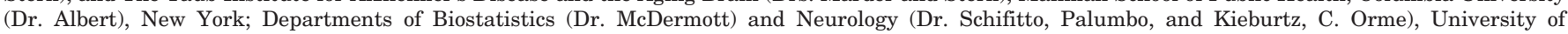

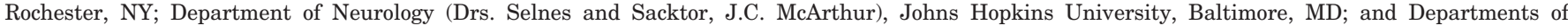
Neurology (Dr. Cohen) and Pediatric Neurology (Dr. Epstein), Northwestern University, Evanston, IL.

Supported by the following NIH grants: NS36519, NS26643, RR00522, RR00044, RR00645.

Received September 16, 2002. Accepted in final form January 27, 2003.

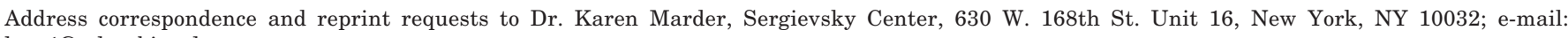
ksm1@columbia.edu 
Table 1 Clinical staging of HIV dementia: NEAD classification

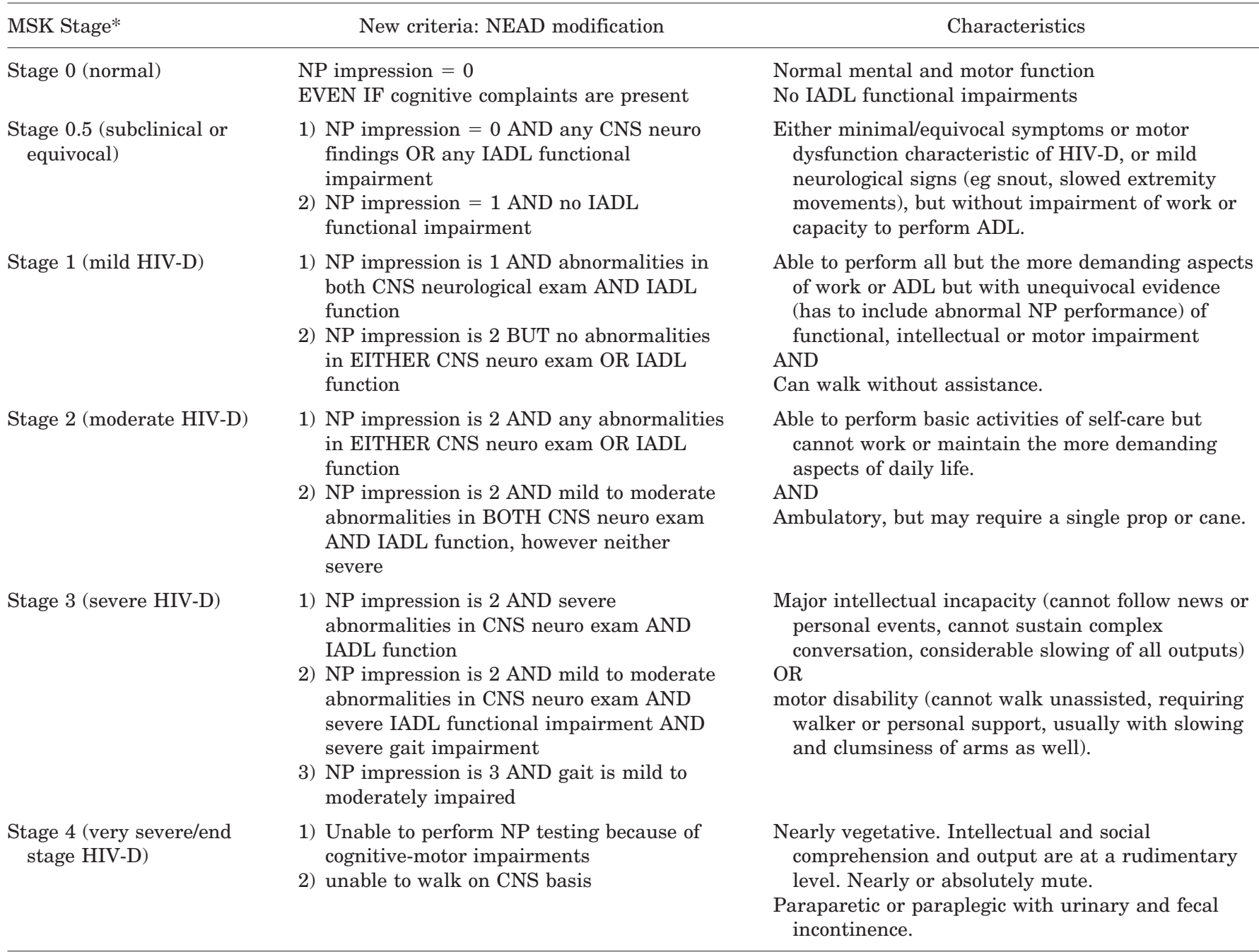

NP impression may be the neuropsychologist's global cognitive impression or the quantitative global neuropsychological impression.

* Developed at Memorial Sloan Kettering Center. ${ }^{8}$

$\mathrm{ADL}=$ activities of daily living; IADL = instrumental activities of daily living.

cal evidence of schizophrenia, and 3) history of chronic neurologic disorders such as MS or uncontrolled epilepsy. Current alcohol or drug use were not grounds for exclusion.

Assessments. Clinical assessments were performed every 6 months for up to 48 months. At each visit, subjects underwent a medical history, a standardized neurologic examination, a neuropsychological battery, functional and psychiatric assessments, and a blood draw for laboratory studies including viral load and immune activation markers. CSF was collected every 12 months.

Neurologic examination. The neurologic examination was designed to detect signs associated with HIV-D. The macro-neurologic examination created for the AIDS Clinical Trials Group and the motor subscale (Part III) of the Unified PD Rating Scale $(\mathrm{UPDRS})^{9}$ were performed by examiners who were videotaped to ensure reliable administration, after reviewing a teaching tape. Items specifically men- tioned in the MSK staging (e.g., snout, slowed extremity movements) (table 1) as well as other signs indicative of either minimal or unequivocal motor dysfunction were used to derive the neurologic scores used in the new MSK algorithm.

Neuropsychological examination. The neuropsychological battery was designed to delineate HIV-D and MCMD. The eight tests included in the core neuropsychological battery covered six domains. Verbal memory was assessed with the Rey Auditory Verbal Learning Test. ${ }^{10}$ Visual memory was assessed with the Rey-Osterrieth Complex Figure Delayed Recall Test. ${ }^{10}$ Constructional skills were assessed with the Rey-Osterrieth Complex Figure Immediate Recall Test. ${ }^{10}$ Psychomotor skills were measured with the Digit Symbol Test. ${ }^{11}$ Motor skills were assessed with the Grooved Pegboard (dominant and nondominant hand $)^{12}$ and timed gait tests. Frontal systems were assessed with Verbal Fluency ${ }^{13}$ and the OddMan-Out tests. ${ }^{14,15}$ Reaction time was measured with 
Table 2 Algorithm for determining quantitative global impression of cognitive function

\begin{tabular}{lll}
\hline NP domain & \multicolumn{1}{c}{ Test } & \multicolumn{1}{c}{ Subtest } \\
\hline Verbal memory & $\begin{array}{l}\text { Rey Auditory Verbal } \\
\text { Learning Test }\end{array}$ & $\begin{array}{l}\text { Trial 5 } \\
\text { Delayed recall } \\
\text { Delayed recognition } \\
\end{array}$ \\
Visual memory & Rey Complex Figure & Delayed recall \\
Visuoconstruction & Rey Complex Figure & Copy \\
Frontal/executive & Odd-Man-Out & (Total score) \\
& Verbal fluency & (Total score) \\
Psychomotor & Digit symbol & (Total score) \\
& CALCAP & Choice \\
& & Sequential \\
Motor speed & Grooved pegboard & Dominant \\
& & Nondominant
\end{tabular}

A score $\geq 1$ SD below the appropriate norm earns 1 point, and a score $\geq 2$ SDs below the appropriate norm earns 2 points; thus, the range of the summary impairment score is $0-24$. A summary impairment score of $(0-1)=$ quantitative global impression of 0 (normal); (2-6) = quantitative global impression of 1 (mild); $(7-14)=$ quantitative global impression of 2 (moderate); and $(15-24)=$ a quantitative global impression of 3 (severe).

$\mathrm{NP}=$ neuropsychological; CALCAP $=$ California Computerized Assessment Package.

the California Computerized Assessment package (CALCAP). ${ }^{16}$ Scores for subjects with $\leq 12$ years of education were compared with norms established by the AIDS Link to IV Experience (ALIVE) ${ }^{17}$ cohort, whereas scores of subjects with $>12$ years of education were compared with norms established by the Multicenter AIDS cohort study. ${ }^{18}$ Cognitive impairment sufficient to meet study enrollment criteria was defined as performance 2 SDs below the appropriate mean on one test, or $1 \mathrm{SD}$ below the mean on two tests. If timed gait was the only measure on which subjects scored 2 SDs below the mean, subjects were not considered to have met cognitive impairment criteria.

The neuropsychological battery was administered at each site by a trained neuropsychometrician. The neuropsychologist at each site assigned a global neuropsychological impression (normal, mild, moderate, or severe) to each subject. The exception was for subjects at the Northwestern site, for whom this global impression rating was assigned by the neuropsychologist at Johns Hopkins because there was no participating neuropsychologist at the Northwestern site. For this rating, a summary of individual neuropsychological subtest raw scores as well as age and education adjusted z-scores for each subject was used. The rating was based on the overall gestalt of the test scores, taking into account specific factors such as the estimated pre-morbid IQ (NART), as well as the number and specific cognitive domains impaired for each subject. For all sites, the rating was based on the actual neuropsychology test scores without any interaction with the subject.

In addition, a separate algorithm was created to generate a quantitative global neuropsychological impression (table 2). Eight tests yielded 12 subtest scores that were used to generate the quantitative neuropsychological rating (see table 2).

Functional measures. Functional measures were chosen that would reflect the degree to which cognitive deficits compromised everyday function, a requirement for the diagnoses of HIV-D and MCMD. Measures were derived from the Instrumental Activities of Daily Living (IADL) scales of Lawton and Brody, ${ }^{19}$ the Katz Instrumental Activities of Daily Living/Lawton Personal Self-Maintenance Scale, ${ }^{20}$ and the role functioning items of the Medical Outcomes Study. ${ }^{21}$ Two functional outcomes that reflect stress and stamina were also included: the Karnofsky performance scale $^{22}$ and the Medical Outcomes Study physical function subscale. ${ }^{21}$ To meet the minimal functional deficit criterion for the MSK staging, respondents were required to report deficits in at least one of the eight IADL (e.g., using telephone, handling money, taking medication, doing laundry, light housework, preparing meals, shopping for groceries, getting to places beyond walking distance).

Psychiatric assessment. The Beck depression inventory ${ }^{23}$ was administered to assess depressive symptoms. This is a 21-item self-report scale that is considered the standard to which other self-report depression scales are compared. Depression items are not included in the MSK; however, in forming their global neuropsychological impression, neuropsychologists considered the impact of depression on cognitive impairment.

Modification of the MSK rating system. Over a 3 -year period, investigators in the NEAD Consortium met three times to determine which items from the clinical assessments would be included in the modified MSK staging, and to determine how the assessments would be used to assign an MSK stage. For example, it was decided that peripheral neuropathy would not be considered as a neurologic abnormality in the MSK rating whereas myelopathy and any CNS abnormality would. Specific scores on each of the items were chosen as cutoffs for defining abnormalities.

In the neurologic domain, a three-tier categorization was developed (no, mild, or severe abnormality) based on the neurologist's evaluation of oculomotor, motor, and extrapyramidal signs (EPS). Twelve CNS neurologic signs were included within these three larger categories.

"Normal" status required that subjects show no deficits in ocular motility (smooth pursuit, saccades), strength and coordination, and reflexes and no EPS. "Mild" status assignment required "mild to moderate abnormality" on any one of the same indicators. "Severe" status required that subjects meet criteria for extrapyramidal deficits along with deficits in ocular motility or motor signs. Subjects met the EPS criterion if they had any abnormality on the UPDRS. Subjects met the motor domain criterion if they scored in the abnormal range in upper or lower ex- 
tremity strength or coordination. Subjects met the oculomotor criterion if they showed abnormalities in smooth pursuit or saccades.

A similar approach was developed for the function and ambulation domains. For example, mild functional deficit required that subjects report a need for help with no more than two household tasks or at least one of the broad role competency indicators (i.e., report of inability to perform tasks carefully). Severe functional deficit required that subjects report a need for help with three or more household tasks. Functional impairment had to be attributed to CNS rather than peripheral nervous system (PNS) abnormalities. At the investigator meetings that led to the development of the algorithm, each of the sites examined data from randomly selected cases and modifications to the rating system were made based on discussions of disagreements. This process continued until all sites were rating cases similarly and investigators agreed upon a formal algorithm for assigning MSK stages (see table 1).

Subjects were assigned an MSK stage according to severity of deficit in four dimensions: neuropsychological performance, neurologic status, self-rated function, and ambulation. For example, one challenge was to differentiate "normal" (MSK 0), "subclinical or equivocal" deficit (MSK 0.5), and "mild HIV-D" (MSK 1). Subjects were assigned MSK 0 if their cognitive performance was in the normal range and they also had no deficits in neurologic, functional, or ambulation status. Subjects were assigned MSK 0.5 if they had no or mild cognitive impairment but abnormalities in either the neurologic or functional domain. Subjects were assigned MSK 1 if they had mild cognitive impairment and abnormalities in both neurologic and functional domains, or if they had moderate cognitive impairment but no other abnormalities. In this way, MSK scores were consistently assigned based on combinations of severity across the four dimensions. These distinctions emerged from our understanding of the original scale, clinical experience regarding the significance of the deficit types, and the empirical co-occurrence of deficits in the cohort.

Design of the inter-rater reliability study. The data necessary to arrive at an MSK rating, from the neurologic, neuropsychological, and functional assessments, were obtained from 25 NEAD cohort subjects at each of the four sites (100 patients in all). All data were collected on standard case report forms at each site and abstracted directly from these case report forms onto a standardized single-page form by individuals who were not involved in the rating process. These forms were sent to the central data coordination center (Rochester, NY) where subject and site identifiers were removed, and the complete set of 100 forms was sent to each of the four sites. At each site the investigators, including at least one neurologist and one neuropsychologist, provided consensus MSK ratings based on the abstracted data and the algorithm (see table 1). Two ratings for each form were provided; one that used the neuropsychologist's global impression of cognitive functioning that was directly abstracted onto the data form, and a second that instead used the quantitative global impression of cognitive functioning (see table 2).

In addition, a SAS program (SAS Institute, Inc., Cary NC) was written to implement the algorithm in table 1 so that a modified MSK rating could be computer-generated from the information in the NEAD database. To classify individual subjects, the information in the database was grouped into four domains: neurologic, neuropsychological (the quantitative global impression of cognitive functioning derived in table 2), functional, and mobility. The degree of abnormalities in each domain was used to compute the MSK rating. Whether functional or mobility impairments were due to CNS or peripheral nervous system (PNS) abnormalities was not captured in the computerized ratings.

Statistical analysis. Weighted kappa statistics ${ }^{24}$ were used to assess the inter-rater reliability for all six possible pairs of sites for each of the two MSK ratings that were generated. Weighted kappa statistics were also used to assess the agreement between each site's MSK rating (using the quantitative global impression of cognitive functioning) and the computergenerated MSK rating.

Results. The percentages of individuals rated at each stage according to the abstracted global NP impression and the quantitative global neuropsychological impression at each site are shown in table 3. Intersite agreement for MSK ratings is shown in table 4. Weighted kappa values are in the good to excellent range ${ }^{24}$ with the majority being in the excellent range $(>0.75)$. In general, investigators at Columbia and Northwestern rated subjects as slightly less impaired than investigators at Johns Hopkins and Rochester. The weighted kappa values were similar for MSK ratings derived from the neuropsychologist's global impression and the quantitative neuropsychologist's impression. The agreement between computer-generated MSK scores and clinician consensus ratings at each of the sites was also in the good to excellent range.

Discussion. We have shown that by operationalizing the criteria proposed in the MSK, first described in 1988 as the "Clinical Staging of the AIDS Dementia Complex,"4 we were able to achieve good to excellent reliability among investigators at four sites using either the neuropsychologist's global impression of cognitive function or a quantitative neuropsychological impression based solely on test scores. We then demonstrated good to excellent reliability between a completely computerized algorithm that included a quantitative neuropsychological impression and the MSK ratings at each site.

Although scores must be reviewed to eliminate impairments that clearly predate HIV infection, the creation of a computerized algorithm may be useful in epidemiologic studies and clinical trials, particularly those that involve multiple sites, providing an outcome measure that is less encumbered by individual judgments by investigators. However, the algorithm is only as good as the data collected, and the presumption that standardized assessments are being performed is implicit in determining whether 
Table 3 Percentages of individuals at each site rated at each MSK stage using Abstracted NP impression and calculated NP impression

\begin{tabular}{|c|c|c|c|c|}
\hline Site & Columbia, $\%$ & Johns Hopkins, \% & Rochester, \% & Northwestern, \% \\
\hline \multicolumn{5}{|c|}{ Abstracted Global NP Imp } \\
\hline MSK 0 & 20.7 & 11.0 & 12.7 & 26.8 \\
\hline MSK 0.5 & 43.9 & 44.0 & 49.4 & 41.5 \\
\hline MSK 1 & 26.8 & 23.0 & 24.0 & 23.2 \\
\hline MSK 2 & 8.5 & 18.0 & 11.4 & 7.3 \\
\hline MSK 3 & 0 & 4.0 & 2.5 & 1.2 \\
\hline \multicolumn{5}{|c|}{ Quantitative Global NP Imp } \\
\hline MSK 0 & 16.2 & 9.0 & 9.5 & 21.0 \\
\hline MSK 0.5 & 39.4 & 42.0 & 42.1 & 36.0 \\
\hline MSK 1 & 22.2 & 19.0 & 20.0 & 24.0 \\
\hline MSK 2 & 21.2 & 26.0 & 24.2 & 18.0 \\
\hline MSK 3 & 1.0 & 4.0 & 4.2 & 1.0 \\
\hline
\end{tabular}

MSK = Memorial Sloan Kettering; NP = neuropsychological.

this exercise translates into true reliability in the field. We have tried to ensure standardized administration of all aspects of the exams by using training manuals, videotapes, and frequent case discussions among examiners to hone definitions. This study did not assess reliability among examiners based on actual patient observation, testing, and scoring. Rather, the study assessed the reliability of determining clinical stage of cognitive impairment based on abstracted data. It may also be difficult in a computerized algorithm to capture the nuances in mobility. While individuals with distal sensory polyneuropathy were not

Table 4 Weighted kappa and percent agreement for MSK ratings

\begin{tabular}{lcc}
\hline Abstracted global NP impression & $\begin{array}{c}\text { Weighted } \\
\text { kappa }\end{array}$ & $\begin{array}{c}\text { Percent } \\
\text { agreement }\end{array}$ \\
\hline Columbia-Johns Hopkins & 0.79 & 76.8 \\
Columbia-Univ Rochester & 0.78 & 75.8 \\
Columbia-Northwestern & 0.81 & 78.8 \\
Univ Rochester-Johns Hopkins & 0.87 & 86.3 \\
Univ Rochester-Northwestern & 0.74 & 71.6 \\
Johns Hopkins-Northwestern & 0.71 & 69.0 \\
Quantitative global NP impression & & \\
Columbia-Johns Hopkins & 0.74 & 78.0 \\
Columbia-Univ Rochester & 0.73 & 76.9 \\
Columbia-Northwestern & 0.77 & 76.3 \\
Univ Rochester-Johns Hopkins & 0.91 & 92.4 \\
Univ Rochester-Northwestern & 0.74 & 73.4 \\
Johns Hopkins-Northwestern & 0.70 & 70.7 \\
Site vs computer-generated & & \\
Computer-Columbia & 0.70 & 67.0 \\
Computer-Johns Hopkins & 0.77 & 75.3 \\
Computer-Univ Rochester & 0.79 & 75.5 \\
Computer-Northwestern & 0.62 & 56.7 \\
\hline
\end{tabular}

$\mathrm{NP}=$ neuropsychological. considered neurologically impaired according the algorithm, the contribution that the neuropathy made to gait and functional impairment could not be completely captured in the algorithm.

It is also clear that even with experienced investigators an intensive effort is required to test and refine definitions when using categorical and subjective scales. Only after three full-day meetings over a 3-year period were the investigators interpreting non-quantified or semi-quantified ratings similarly. At that time, table 1 was created to reflect the collective thinking of the investigators. A computerized algorithm cannot substitute for this ongoing training, and should be employed only when consensus has been achieved.

Inter-rater reliability for diagnosis of dementia. Inter-rater reliability of clinical diagnostic criteria for a number of neurodegenerative dementing disorders has been examined. The use of clinical diagnostic criteria for neurodegenerative diseases has improved inter-rater reliability and accuracy in clinicopathological studies. The American Psychiatric Association diagnostic criteria from the Diagnostic and Statistical Manual (DSM III-R), ${ }^{25}$ National Institute of Neurological Disorders and Stroke-ADRDA, ${ }^{26}$ the Eisdorfer and Cohen Research Diagnostic Criteria (ECRDC), and ICD-10 diagnostic criteria for $\mathrm{AD}$ have been assessed separately ${ }^{27,28}$ and compared. ${ }^{29,30}$ Inter-rater reliability of the Lund Manchester criteria for frontotemporal dementia ${ }^{31}$ the Consensus Guidelines for the Clinical Diagnosis of Dementia with Lewy Bodies (DLB) ${ }^{32,33}$ and the National Institute of Neurologic Disorders and Stroke (National Institute of Neurological Disorders and Stroke) and the Association Internationale pour la Recherche et l'Enseignement en Neurosciences (AIREN) ${ }^{34}$ diagnostic criteria for vascular dementia has been assessed. In general, when data are abstracted and the same information is provided to raters with similar training, and research diagnostic criteria are applied for 
specific diseases, kappa statistics are in the good to excellent range. Inter-rater reliability may be decreased in situations where a second potential cause of dementia exists (e.g., $\mathrm{AD}$ and vascular disease) ${ }^{34}$ and modest for Dementia with Lewy bodies, ${ }^{31}$ perhaps because of the overlap in clinical criteria with $\mathrm{AD}$.

Inter-rater reliability in HIV-related studies. Although several quality of life measures have been shown to be reliable and valid in studies of HIVinfected individuals, ${ }^{35,36}$ little has been published on the reliability of the neurologic examination or neurologic rating scales. Inter-rater reliability was assessed among three experienced neurologists on 20 subjects participating in a natural history study of HIV-infected individuals. ${ }^{37}$ Reliability was in the good-to-excellent range for examination of mentation and cranial nerve function and in the fair-to-good range for pyramidal tract signs, cerebellar signs, sensory, and extrapyramidal signs. Overall agreement (intraclass correlation) on a summary measure of neurologic function, the modified Kurtzke score, was 0.89 . The inter-rater agreement of a modification of the Karnofsky Performance scale was assessed in 657 HIV-infected individuals, and found to be strong (0.82) between two physicians and slightly less between a nurse and a physician (0.77). ${ }^{38}$

The importance of continued training and consensus building in creating an algorithm for staging HIV-D cannot be underestimated. This has led to the development of a computerized algorithm that may prove useful in multicenter clinical trials and observational studies of $\mathrm{HIV}$-associated cognitive impairment. A reliable instrument is necessary for future clinicopathologic studies in which diagnostic accuracy will be assessed.

\section{Appendix: Members of the NEAD Consortium}

Columbia University: Karen Marder, MD, MPH; Yaakov Stern, PhD; Steven Albert, PhD; George Todak, MSW; Ronda Clouse, RN; Jose Beltre, BA; Ivan Simonetti, BA. Johns Hopkins University: Ned Sacktor, MD; Ola Selnes, PhD; Deneen Esposito, BA; Ruby Agoha, BA; David Burgess, MA; Jason Creighton, BA; Justin McArthur, MBBS, MPH. University of Rochester: Giovanni Schifitto, MD; Michael McDermott, PhD; Donna Palumbo, $\mathrm{PhD}$; Connie Orme, BA; Larry Preston, BPS; Keith Bourgeois, BS; Kim Cruttenden, MS; Karl Kieburtz, MD, MPH. Northwestern University: Leon G. Epstein, MD; Bruce A. Cohen; Linda Reisberg, RN.

\section{References}

1. The Dana Consortium on Therapy for HIV Dementia and Related Cognitive Disorders. Clinical confirmation of the American Academy of Neurology algorithm for HIV-1-associated cognitive/motor disorder. Neurology 1996;47:1247-1253.

2. Janssen RS, Cornblath DR, Epstein LG, et al. Nomenclature and research case definitions for neurological manifestations of human immunodeficiency virus type-1 (HIV-1) infection. Report of a Working Group of the American Academy of Neurology AIDS Task Force Neurology 1991;41:778-785.

3. Stern Y, McDermott MP, Albert S, et al. Factors associated with incident human immunodeficiency virus-dementia. Arch Neurol 2001;58: 473-479.

4. Price RW, Brew BJ. The AIDS dementia complex. J Infect Dis 1988;158: 1079-1083.

5. The Dana Consortium on Therapy for HIV Dementia and Related Cognitive Disorders. A randomomized, double-blind, placebo-controlled trial of deprenyl and thioctic acid in human immunodeficiency virusassociated cognitive impairment. Neurology 1998;50:645-651.

6. The Dana Consortium on Therapy for HIV Dementia and Related Cognitive Disorders. Safety and tolerability of the antioxidant OPC-14, 117in HIV associated cognitive impairment. Neurology 1999;49:142146.

7. Schifitto G, Sacktor N, Marder K, et al. Randomized trial of the platelet-activating factor antagonist lexipafant in HIV-associated cognitive impairment. Neurological AIDS Research Consortium. Neurology 1999;53:391-396.

8. Sacktor N, Schifitto G, McDermott MP, Marder K, McArthur JC, Kieburtz K. Transdermal selegiline in HIV-associated cognitive impairment: pilot, placebo-controlled study. Neurology 2000;54:233-235.

9. Fahn S, Marsden C, Calne D. Recent Developments in Parkinson's disease. Florham Park: Macmillan Healthcare Information, 1987.

10. Rey A. L'examen psychologique dans les cas d'encephalopathie traumatique. Arch Psychologie 1941;28:286-340.

11. Wechsler D. Wechsler Adult Intelligence Scale-Revised. New York: The Psychological Corporation, 1981.

12. Klove H. Clinical neuropsychology. Med Clin North Am 1963;46:16471658.

13. Benton AL, Hamsher KD. Multilingual Aphasia Examination. Iowa City: University of Iowa, 1976.

14. Flowers KA, Robertson C. The effects of Parkinson's disease on the ability to maintain a mental set. J Neurol Neurosurg Psychiatry 1985; 48:517-529.

15. Richards M, Cote LJ, Stern Y. Executive function in Parkinson's disease: set-shifting or set- maintenance? J Clin Exp Neuropsychol 1993; 15:266-279.

16. Miller EN, Satz P, Visscher B. Computerized and conventional neuropsychological assessment of HIV-1 infected homosexual men. Neurology 1991;41:1608-1616.

17. Selnes OA, Jacobson L, Machado AM, et al. Normative data for a brief neuropsychological screening battery. Percept Mot Skills 1991;73:539_ 550 .

18. Concha M, Selnes OA, McArthur J, et al. Normative data for a brief neuropsychologic test battery in a cohort of injecting drug users. Intl $\mathrm{J}$ Addictions 1995;30:823-841.

19. Lawton MP, Brody EM. Assessment of older people: self-maintaining and instrumental activities of daily living. Gerontologist 1969;9:179186.

20. Katz S, Ford A, Moskowitz R, et al. Studies of illness in the aged: the index of ADL. JAMA 1963;183:914-919.

21. Stewart AL, Ware JE. Measuring function and well-being: The Medical Outcomes Study Approach. Durham: Duke University Press, 1993.

22. Karnofsky DA, Abelman WH, Carver LF, et al. The use of nitrogen mustards in the palliative treatment of carcinoma. Cancer 1948;1:634656.

23. Beck AT, Steer R, Brown J. BDI-II Manual. San Antonio: The Psychological Corp., 1996.

24. Fleiss JL. Statistical Methods for Rates and Proportions. New York: John Wiley \& Sons Inc., 1981

25. American Psychiatric Association. Diagnostic and Statistical Manual of Mental Disorders, rev. 3rd ed. Washington: American Psychiatric Press Inc, 1987.

26. McKhann G, Drachman D, Folstein M, Katzman R, Price D, Stadlan EM. Clinical diagnosis of Alzheimer's disease: report of the NINCDSADRDA Work Group. Neurology 1984;34:939-944.

27. Lopez OL, Swihart AA, Becker JT, et al. Reliability of NINCDS-ADRDA clinical criteria for the diagnosis of Alzheimer's disease. Neurology 1990;40:1517-1522.

28. Blacker D, Albert MS, Bassett SS, Go RC, Harrell LE, Folstein MF. Reliability and validity of NINCDS-ADRDA criteria for Alzheimer's disease. The National Institute of Mental Health Genetics Initiative Archives of Neurology 1994;51:1198-1204.

29. Kukull WA, Larson EB, Reifler BV, Lampe TH, Yerby M, Hughes J. Interrater reliability of Alzheimer's disease diagnosis. Neurology 1990;40:257-260.

30. Baldereschi M, Amato MP, Nencini P, et al. Cross-national interrater agreement on the clinical diagnostic criteria for dementia. WHO-PRA Age-Associated Dementia Working Group, WHO-Program for Research on Aging, Health of Elderly Program. Neurology 1994;44:239-242.

31. Lopez OL, Litvan I, Catt KE, et al. Accuracy of four clinical diagnostic criteria for the diagnosis of neurodegenerative dementias. Neurology 1999;53:1292-1299.

32. McKeith IG, Fairbairn AF, Bothwell RA, et al. An evaluation of the predictive validity and inter-rater reliability of clinical diagnostic criteria for senile dementia of Lewy body type. Neurology 1994;44:872-877.

33. Mega MS, Masterman DL, Benson DF, et al. Dementia with Lewy bodies: reliability and validity of clinical and pathologic criteria. Neurology 1996;47:1403-1409.

34. Lopez OL, Larumbe MR, Becker JT, et al. Reliability of NINDS-AIREN clinical criteria for the diagnosis of vascular dementia [see comments]. Neurology 1994;44:1240-1245. 
35. Wu A, Rubin H, Mathews W, et al. A health status questionnaire using 30 items from the Medical Outcomes Study. Med Care 1991;29:786798

36. Bozzette SA, Hays RD, Berry SH, Kanouse DE. A Perceived Health Index for use in persons with advanced HIV disease: derivation, reliability, and validity. Med Care 1994;32:716-731.
37. Marder K, Liu X, Stern Y, et al. Neurologic signs and symptoms in a cohort of homosexual men followed for 4.5 years. Neurology 1995;45: 261-267.

38. Fantoni M, Izzi I, Del Borgo C, et al. Inter-rater reliability of a modified Karnofsky Scale of Performance Status for HIV-infected individuals. AIDS Patient Care \& Stds 1999;13:23-28.

\title{
Age at focal epilepsy onset varies by sex and hemispheric lateralization
}

\author{
M.J. Doherty, MD; S. Jayadev, MD; J.W. Miller, MD, PhD; D.F. Farrell, MD; M.D. Holmes, MD; and \\ C.B. Dodrill, $\mathrm{PhD}$
}

\begin{abstract}
Background: Previous studies have shown that interictal epileptiform discharges favor the left hemisphere in adults but the right side in children up until age 5. This may be due to sex-influenced asymmetric brain maturation. To clarify this relationship, the authors analyzed age at epilepsy onset by sex and by lateralization of epileptiform activity. Methods: An adult epilepsy center long-term monitoring database was used to define patients with exclusively unilateral epileptiform findings. Three groups were studied: any epileptiform activity $(\mathrm{n}=404)$, ictal activity $(\mathrm{n}=287)$, and interictal activity $(\mathrm{n}=265)$. The second and third groups were drawn from the first group and the second and third groups overlapped with each other. Side of lateralized finding and sex were analyzed via factorial two-way analysis of variance with the outcome variable being age at epilepsy onset. Comparison analysis included patients with generalized epilepsy $(\mathrm{n}=114)$, nonepileptic seizures (NES, $\mathrm{n}=232$ ), and surgical mesial temporal sclerosis (MTS, $\mathrm{n}=116)$. Results: Patients with unilateral epileptiform activity displayed bimodal epilepsy onset ages with infant and adolescent peaks. For patients with a right-sided focus, epilepsy onset was earlier in men (14.4 years) than women (20.7 years). In contrast, among patients with a left-sided focus, epilepsy began earlier in women (18.2 years) than men (19.9 years, $p<0.01)$. Parallel results were found in unilateral ictal $(p<0.01)$ and unilateral interictal activity $(p=0.01)$. Patients with surgical MTS, NES, or generalized seizure showed no similar patterns. Conclusions: In adult patients with focal epilepsy, sex and lateralized epileptiform abnormalities may be related to age at epilepsy onset.
\end{abstract}

NEUROLOGY 2003;60:1473-1477

In adults, focal interictal epileptiform discharges (ED) show a preference for the left hemisphere. ${ }^{1-4}$ In contrast, children show interictal favoring of right hemispheric ED until age 5, after which left hemispheric ED become more frequent. ${ }^{5}$ Because normal brain maturation and development occurs asymmetrically at differing rates and times, age-related hemispheric favoring of ED might reflect age-specific brain asymmetries. ${ }^{6-10}$ Sex influences brain asymmetry and maturation but has yet to be extensively correlated with lateralization of ED and age at epilepsy onset. ${ }^{11-15}$ In this study we test the hypothesis that among adults with focal epilepsy, age at epilepsy onset varies according to both sex and lateralization of ED.

Methods. We studied 1,215 consecutive first scalp long-term video-EEG monitoring (LTM) database entries. Three exclusively unilateral groups were defined: Group $1(\mathrm{n}=404)$ included patients with ictal or interictal ED. Group $2(\mathrm{n}=289)$ displayed only ictal discharges. Group $3(\mathrm{n}=284)$ demonstrated interictal spike or sharp wave activity. Groups 2 and 3 were subsets of Group 1.
Lateralization of ED was determined by electroencephalographers blind to study objectives. Multifocal unilateral ictal, interictal, or secondarily generalized activities were studied. Combinations of bilateral ictal or interictal activity, including primary generalized activity, were excluded. LTM performed on patients $<18$ years were excluded, as were invasive or follow-up studies.

Group 4 was defined from a separate database: patients diagnosed pathologically with mesial temporal sclerosis (MTS, n = 116). Forty-three Group 4 patients overlapped with Group 1. The LTM database was used to define Group $5(\mathrm{n}=114)$ patients with generalized epilepsy, and Group $6(\mathrm{n}=232)$ patients with LTM demonstration of both nonepileptic seizures (NES) and absent ED.

Age at epilepsy onset was defined as the age, in years, during which repeated, spontaneous, nonfebrile seizures first occurred. Statistical testing was performed using SPSS (Chicago, IL). Groups 1 through 3 were analyzed for whether sex and hemispheric lateralization of electrical discharges influenced age at first seizure using two-way factorial analysis of variance (ANOVA). For Group 4, surgical MTS, side of surgical resection was used as the lateralization variable. Supplemental $t$-test analysis on Group 1 by sex or hemispheric abnormality clarified underlying effects not evident from ANOVA. Subsets of Group 1 defined by handedness, a family history of seizure, prior neurologic insult (such as birth injury, head trauma, brain tumor, stroke, meningitis, or encephalitis), or antecedent febrile seizure were analyzed similarly to Groups 1 through 3. Duration between

From the Regional Epilepsy Center and Departments of Neurology (Drs. Doherty, Jayadev, Miller, Farrell, Holmes, and Dodrill) and Neurological Surgery (Drs. Miller and Dodrill), University of Washington, Seattle.

Received October 21, 2002. Accepted in final form January 31, 2003.

Address correspondence and reprint requests to Dr. M.J. Doherty, Department of Neurology, University of Washington, Box 356465, Seattle, WA 98195; e-mail: mdoherty@u.washington.edu 


\section{Neurology}

\section{Inter-rater reliability of a clinical staging of HIV-associated cognitive impairment}

K. Marder, S. M. Albert, M. P. McDermott, et al.

Neurology 2003;60;1467-1473

DOI 10.1212/01.WNL.0000064172.46685.82

\section{This information is current as of May 13, 2003}

\section{Updated Information \& \\ Services}

References

Citations

Subspecialty Collections

Permissions \& Licensing

Reprints including high resolution figures, can be found at:

http://www.neurology.org/content/60/9/1467.full.html

This article cites 31 articles, 16 of which you can access for free at: http://www.neurology.org/content/60/9/1467.full.html\#\#ref-list-1

This article has been cited by 9 HighWire-hosted articles: http://www.neurology.org/content/60/9/1467.full.html\#\#otherarticles

This article, along with others on similar topics, appears in the following collection(s):

\section{Clinical trials Methodology/study design}

http://www.neurology.org//cgi/collection/clinical_trials_methodology_ study_design_

HIV

http://www.neurology.org//cgi/collection/hiv

HIV dementia

http://www.neurology.org//cgi/collection/hiv_dementia

Information about reproducing this article in parts (figures,tables) or in its entirety can be found online at:

http://www.neurology.org/misc/about.xhtml\#permissions

Information about ordering reprints can be found online: http://www.neurology.org/misc/addir.xhtml\#reprintsus

Neurology ${ }^{\circledR}$ is the official journal of the American Academy of Neurology. Published continuously since 1951, it is now a weekly with 48 issues per year. Copyright. All rights reserved. Print ISSN: 0028-3878. Online ISSN: 1526-632X.

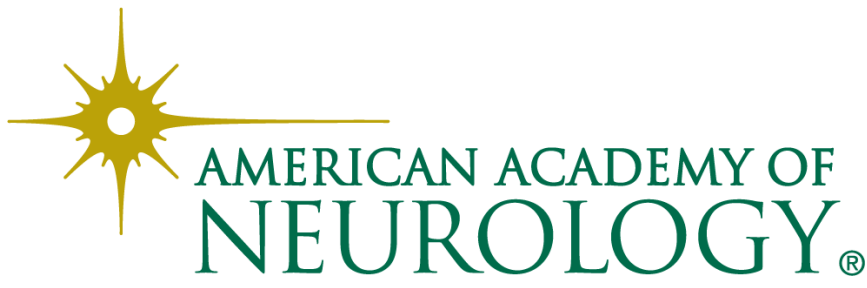

